\title{
Las Decisiones de Financiamiento de Capital
}

\author{
Beatriz Herrera García \\ Facultad Ciencias Contables de la UNMSM \\ Lima-Perú
}

\begin{abstract}
RESUMEN
Las decisiones de financiamiento de capital es encontrar proyectos de inversión cuya rentabilidad supere el coste de llevarlos a cabo, es decir, proyectos que aporten valor a la empresa. El principal problema, dejando a un lado el de la determinación del coste de oportunidad del capital de una decisión de inversión, es el de la valoración del activo que se crearía al realizar la inversión. Así, cuando valoramos una decisión de inversión realizamos una prevision de los flujos de fondos que promete generar en el futuro y procedemos a calcular su valor actual con objeto de poder comparar, en un momento determinado de tiempo (el actual) el valor global de dichos flujos de fondos con respecto al desembolso inicial que implica la realización de dicha decisión de inversión.

Uno de los criterios de comparación más comúnmente empleados en las empresas (Graham and Harvey, et., al.,) es el valor actual o presente neto que además, es el criterio más acorde al objetivo general de todo equipo directivo; la maximización del valor de la empresa, puesto que indica exactamente cuanto se prevé que aumente el valor de una empresa si realiza una decisión de inversión que se esta valorando.

Este criterio considera efectuable una decisión de inversión cuando el valor actual neto es positivo (VAN $>0$ ), es decir, cuando la totalidad de los flujos de fondos esperados descontados a una tasa apropiada al riesgo de la decisión de inversión supera al coste de realizarlo. Por el contrario, si el valor actual neto fuese negativo (VAN $<0)$, sería desaconsejable realizar la decisión de inversión.
\end{abstract}

Palabras clave: Flujo de fondos, valor actual neto

\section{INTRODUCCIÓN}

El nuevo ámbito propiciado por la desregulación financiera ha permitido un notable incremento de la movilidad internacional de capitales, facilitando un mejor aplicación de los recursos a escala mundial, un mayor diversificación de riesgos y en un estímulo al crecimiento de las economía s de los mercados emergentes. Por otro lado, el ahorro de los países desarrollados ha podido obtener mayores rendimientos que los que habrían podido obtener en los mercados financieros de origen. Por otro lado, a todo esto es atribuible una mayor inestabilidad de los mercados de capitales al proceso de regulación financiera. Esta afirmación cierta en parte, en la medida que nos e puede negar su contribución a una fluctuaciones excesivas del mercado, también, es de recordar que estos efectos son debidos a determinadas políticas macroeconómicas (políticas presupuestarias expansivas).

El premio Nobel Merton Millar, afirma que este desarrollo ha sido impulsado por la introducción de instrumentos financieros que redistribuyen el riesgo de forma mas eficiente entre los participantes del mercado 
financiero.Entre los efectos más importantes se tiene a:

- El aumento en la volatilidad en los tipos de interés, tipos de cambio, tasas de inflación y precios de los activos financieros.

- Los avances producidos en la informática y las telecomunicaciones.

- La mayor cultura financiera existente entre los participantes profesionales del mercado.

- La competencia entre los intermediarios financieros.

- Los incentivos para sortear la normatividad legal vigente.

- Los cambios habidos en los modelos globales del bienestar financiero.

De otro lado, la falta de estabilidad en le sistema de cambios, en los tipos de interés, en los mercados, en la solvencia de los países y en resumen, un mayor riesgo en el conjunto de operaciones financieras y comerciales. Muchas empresas se han dado cuenta que esta inestabilidad puede causarles dificultades en la consecuciones de los flujos de fondos previstos, y en algunos casos llevarlos a tomas de control societarios hostiles.

Entre los riesgos a los que esta expuesta una empresa podemos destacar:

- Movimientos en los precios de las materias primas.

- Variaciones en los tipos de cambios de las divisas en las que se denominan dichas materias primas.

- Oscilaciones en le precio de la energía, que se necesita para procesar dichas materias.
- Cambios en el tipo de cambio de su propia moneda (si aumenta, reducirá su competitividad en el exterior, o sufriendo lo contrario si desciende).

- Cambios en las tasas de interés internas, que afectarán al coste de su endeudamiento $\mathrm{y}$, posiblemente, a sus ingresos por ventas.

- Alteraciones en los tipos de interés externos, que afectará a sus competidores $\mathrm{y}$, por lo tanto, al comportamiento de las ventas de la empresa.

Cada una de esta influencia puede ser resumida a través del denominado perfil del riesgo, que identifica y mide el riesgo financiero. Ante estos nuevos y mayores riesgos las empresas tratan en primer lugar de examinar su propia estructura y las características de sus competidores intentando identificar aquellos riesgos que mas pueden afectarles. Esta actitud se puede materializar de dos formas:

a) Cambiando el tipo de operaciones que realizan la empresa, por ejemplo, fusionándose con otra que tenga perfil de riesgo distinto; o

b) Añadiendo a su cartera alguna operación financiera que cubra los posibles riesgos como el uso de productos financieros de los denominados fuera de balance, que permiten a la empresa dejar sus operaciones intactas mientras la protegen de las fluctuaciones en su ambiente. Debido a que el coste de la primera es bastante mas grande que el de la segunda, las empresas tienden cada vez mas a utilizar esta última. 


\section{MARCOTEÓRICO}

En los últimos 25 años, el comercio mundial a crecido al doble que la producción mundial; la inversión extranjera directa tres veces mas rápido; y la negociación transfronteriza en activos financieros diez veces mas veloz.

Por su parte, el desarrollo tecnológico ha permitido conectar los mercados entre si a través de unos sistemas avanzados de informática y telecomunicaciones, posibilitando que los inversionistas e intermediarios financieros estén al tanto de lo que ocurre en los mercados internacionales, lo que les permite valorar el impacto de la información recibida en el comportamiento de sus carteras de valores.

Por otro lado, si hay algún coste que se ha derrumbado en los últimos tiempos éste es el de las telecomunicaciones y el del proceso de datos, permitiendo a las compañías localizar diferentes partes del proceso de producción en otros tantos países pero manteniendo al mismo tiempo, el control total del proceso productivo.

En cambio, en torno a las nuevas tendencias de la economía y desarrollo, han surgido dos grupos: los optimistas y los pesimistas. Para los primeros, la globalización aumentará la productividad y la calidad de vida de las personas y los países. Se basan en que unas economías integradas favorecerán una mejor división del trabajo entre los estados, permitiendo que aquellos que tengan salarios bajos se especialicen en tareas donde la mano de obra sea vital, mientras los que tengan salarios altos utilicen a sus trabajadores en tareas más productivas, según el criterio de ventaja comparativa (Herrera, B. 1988).
Todo ello permitirá a las empresas beneficiarse de las economías de escala subyacentes. Además, los recursos financieros se dirigirán hacia aquellos países que proporciones las oportunidades de inversión más productivas abandonando aquellas zonas donde los rendimientos esperados sean más reducidos. En cambio, el otros grupo cree que el aumento de la competencia de países de salarios bajos destruirá el empleo y empujará los sueldos a la baja en los países desarrollados. Además, los gobiernos no podrán diseñar las políticas económicas que deseen, sino que tendrán que ajustarse a la poca flexibilidad que le permitirá el mercado global junto con el poderoso mercado financiero internacional, siempre proclive a causar estragos y sobresaltos a lo largo y ancho de la economía mundial.

Ambos puntos de vista tienen algo de razón por que efectivamente la mayor competencia mundial puede beneficiar a las personas individualmente (productos de mejor calidad al mejor precio posible, mejores servicios, mayor rentabilidad de las inversiones aun mejor riesgo, etc.), pero para que beneficie al país en su totalidad deberá aplicarse una serie de medidas correctivas que le adapten continuamente a los cambios de tendencia de la economía mundial a base de mejorar su propia productividad y competitividad.

Por otro lado, al derribarse las barreras a los flujos monetarios e integrarse a los mercados financieros ${ }^{1}$, en teoría, unos flujos de capitales mas grandes deberían implicar unos beneficios mayores puesto que el ahorro y la inversión deberían asignarse de una forma mas eficiente. Los países en desarrollo ya no tendrían falta de dinero para acometer las inversiones 
necesarias para su desarrollo económico. Los inversionistas podrían colocar su dinero en cualquier decisión de inversión que les proporciones un rendimiento interesante sin tener que ceñirse a su propio país $\mathrm{y}$, además, el riesgo estaría diversificado al máximo al poder formarse carteras de inversión con casi cualquier decisión de inversión que deseen y en cualquier país (cuanto mayor sea el abanico de posibles inversiones más se diversificará el riesgo).

\section{MÉTODOS Y RESULTADOS}

Unos de los criterios de comparación más comúnmente empleado en las empresas es el VAN, criterio acorde al objetivo general de la corporación: Maximización de la riqueza de la empresa. Su ecuación general es la siguiente:

$$
V A N=-A+\sum_{j=1}^{j-n}\left|\frac{F C:}{(1+k)^{\prime}}\right|
$$

donde:

A Representa el desembolso inicial del proyecto

FC. Representa los diversos flujos de caja esperados

n Es el horizonte temporal del proyecto

$\mathrm{k} \quad$ Es la tasa de descuento (el coste de oportunidad del capital) apropiada al riesgo del proyecto (Footnotes)

Sin embargo, en necesario tener en cuenta que cuando se analiza un proyecto de inversión bajo la óptica del criterio de evaluación VAN. Se están realizando una serie de supuestos que afectan a los resultados obtenidos.

\section{Los supuestos son:}

a) Los flujos de caja que el proyecto promete generar pueden reemplazarse por sus valores medios y éstos se pueden tratar como valores conocidos desde el principio del análisis. Este supuesto implica ignorar que el equipo directivo pueda alterarlos al adaptar su gestión a las condiciones imperantes en el mercado durante toda la vida del proyecto. Esta flexibilidad operativa aporta valor al proyecto de inversión, valor que el método VAN, por ejemplo, es incapaz de reflejar.

b) La tasa de descuento es conocida y constante, dependiendo únicamente del riesgo del proyecto. Los que implica suponer que el riesgo es constante, suposición falsa en la mayoría de los casos, puesto que el riesgo depende de la vida que le quede al proyecto y de la rentabilidad actual del mismo a través del efecto del apalancamiento operativo. Por tanto, la tasa de descuento varia con el tiempo $y$, por tanto es incierta. Ejemplo, en una gran variedad de proyectos de inversión el riesgo disminuye con el tiempo porque conforme el proyecto se va desarrollando el equipo directivo tiene mas información sobre él y es capaz de prever con mayor exactitud los flujos de caja que quedan por recibir, además de aprovecharse de aquella para intentar que estos sean los mas altos posibles.

c) La necesidad de proyectar los precios esperados a lo largo de todo el horizonte temporal del proyecto es algo imposible o temerario en algunos sectores, porque

En cambio, si se tratara de medir el nivel de integración de los mercados de capitales este consistiría en calcular la relación existente entre el desequilibrio de la balanza de pagos en cuenta corriente y el producto bruto interno de los diversos países para, posteriormente, hallar su valor medio, con arreglo a este índice se puede ver aunque esta aumentando $(2.5 \%-3.0 \%)$ todavía está lejos de alcanzar los niveles de comienzo de siglo (5.0\% - 5.5\%). 
la gran variabilidad de aquellos obligaría a esbozar todos los posibles caminos seguidos por los precios al contado a lo largo del horizonte de planificación. Como esto es muy difícil de hacer, de cara a la aplicación del VAN, arbitrariamente se eligen unos pocos de los muchos caminos posibles.

d) Se supone que los VAN del proyectos son aditivos, lo que no es del todo cierto porque no pueden valorar la serie de activos intangible (la flexibilidad operativa y las interacciones entre proyecto), que llevan incorporados aquellos. Por ejemplo, en una fusión de dos empresas el resultado no es la suma exacta de sus valores individuales, salvo por accidente, debido a los efectos sinérgicos implicados.

Por tanto, para fines del estudio podemos redefinir la regla de decisión del VAN que, recordemos, recomendaba aceptar un proyecto cuando el valor de una unidad de capital era superior o igual a su coste de adquisición e instalación. Esta regla es incorrecta porque ignora el coste de oportunidad de realizar la inversión ahora, recurriendo a la opción de esperar para obtener nueva información. Por lo cual, para que una decisión de inversión sea efectuable el valor de todos los flujos de caja efectuados deberá ser superior a su coste de de adquisición e instalación, al menos, en una cantidad igual al valor de mantener viva la opción de inversión

\section{ANÁLISIS Y DISCUSIÓN}

La posibilidad de realizar una decisión de inversion tiene un gran parecido para adquirir un activo financiero (acción o bono). Ambos implican el derecho pero no la obligación de adquirir un activo pagando una cierta cantidad de dinero en un momento determinado. Las opciones reales son aquellas cuyo activo subyacente es un activo real, por ejemplo, un edificio, un proyecto de inversión, una compañía, una patente, etc.

El valor de las opciones es función de seis variables:

a) El precio del activo subyacente. En la opción financiera indica el precio actual del activo subyacente, mientras que en la opción real indica el valor actual del activo real subyacente, es decir, el valor actual de los flujos de caja que espera genere dicho activo.

El impacto que tiene esta variable sobre el valor de las opciones, la afecta de la siguiente forma: si el precio del activo subyacente aumenta (o disminuye) el valor de la opción de compra también lo hará, lo contrario ocurrirá con la opción de venta.

b) El precio de ejercicio. En la opción financiera indica el precio al que el propietario de la opción pueda ejercerla, es decir, el precio que puede pagar por comprar el activo financiero subyacente, o el precio que le pagarán por venderla. En la opción real indica el precio a pagar por hacerse con el activo real subyacente, es decir, con sus flujos de caja (por ejemplo, en una decisión de inversión será el desembolso inicial), o el precio al que el propietario del activo subyacente tiene derecho a venderla, si la opción es de venta.

El impacto que tiene esta variable sobre el varo de las opciones, la afecta de la siguiente forma: si el precio de ejercicio aumenta (o disminuye) el valor de la 
opción de compra descenderá (o aumentará). El valor de la opción de venta se mueve en el mismo sentido que el precio de ejercicio.

c) El tiempo hasta el vencimiento: tiempo del que dispone su propietario para poder ejercer la opción.

El impacto que tiene esta variable sobre el valor de las opciones, la afecta de la siguiente forma: la posibilidad de posponer una decisión de inversion proporciona a la empresa un tiempo adicional para examinar la tendencia de los acontecimientos futuros reduciendo, al mismo tiempo, la imposibilidad de incurrir en costosos errores debido a que los acontecimientos se han desarrollado en contra de lo previsto. Cuando mayor sea el intervalo de tiempo $(\mathrm{t})$ que se tiene de margen para demorará la decisión final, mayor será la posibilidad de que los acontecimientos se desarrollen de forma favorable aumentando la rentabilidad de la decisión de inversión. Es evidente que si dicho acontecimientos fuesen contrarios a los intereses de decisor, éste renunciaría a realizar la decisión de inversión evitando así una pérdida innecesaria.

d) El riesgo de volatilidad: es la varianza o desviación típica (s), de los rendimientos del activo subyacente. Indican la volatilidad del activo subyacente cuyo precio medio es el precio del activo subyacente, pero que puede oscilar en el futuro, la medida de dicha oscilación es la volatilidad.

El impacto que tiene esta variable sobre el valor de las opciones, la afecta de la siguiente forma: en cuanto al riesgo asociado a la decisión de inversión es preciso señalar que cuanto mas grande sea, más valiosa será la opción sobre la inversión. Ello se debe a la simetría existente entre pérdidas y ganancias; así, un aumento de las operaciones hará aumentar las posibilidad del VAN, mientras que una gran descenso de aquellas no necesariamente hará que el VAN sea negativo (porque, en este caso, se puede eliminar las pérdidas al no ejercer la opción de inversión). Concretando, un aumento del valor de la opción de invertir no significa que aumente el deseo de hacerlo, puesto que aumento del riesgo reduce el deseo de invertir (o retrazar la decisión de inversión), debido a que el incremento en el valor de la decisión de inversión se debe, precisamente, al valor de la espera. Por tanto, el aumento del valor de la opción de inversión refleja exactamente la necesidad de esperar todo lo que se pueda antes de proceder a realizar la decisión de inversión.

e) El tipo de interés sin riesgo, refleja el valor temporal del dinero.

El impacto que tiene esta variable sobre el valor de las opciones, la afecta de la siguiente forma: por la misma razón anterior (el aumento del valor de la opción de inversión refleja exactamente la necesidad de esperar), un aumento del tipo de interés sin riesgo produce un descenso del valor del activo (al penalizar el valor actual de los flujos de caja esperados) y, al mismo tiempo, reduce el valor actual del precio de ejercicio de la opción. Por lo general, pero no siempre, el efecto neto 
resultante induce a pensar que un aumento del tipo de interés sin riesgo provoca un ascenso del valor de las decisiones de inversión con opciones de expansión (esto es, que una aumento del tipo de interés sin riesgo suele reducir con mas fuerza al valor actual de precio de ejercicio que al valor del activo).

f) Los dividendos, dinero líquido generado por el activo subyacente durante el tiempo que el propietario de la opción la posee y no la ejerce. Si la opción es de compra, este dinero lo pierde el propietario de la opción (porque si hablamos de una opción de compra de acciones, mientras ésta no se ejerza su propietario no sería accionista $y$, por tanto, no tendrá derecho a los dividendos). En el caso de las opciones reales de compra, es el dinero que genera el activo subyacente (o al que se renuncia), mientras el propietario de aquella no la ejerza.

Finalmente, el impacto que tiene esta variable sobre el valor de las opciones, la afecta de la siguiente forma: en cuanto a los dividendos, cuanto mayor sea su valor, menor es el valor de la opción de compra. En el contexto de las decisiones de inversión reales, los dividendos vienen a ser los flujos de caja a los que s e renuncia por no haber realizado ya la decisión de inversión. Por ejemplo, si Ud. Está pensando en si lleva a cabo, o no, el desarrollo de una patente, mientras no lo haga, esta dejando de ganar los flujos de liquidez que le proporcionarían el haberlo desarrollado ya.

\section{CONCLUSIONES}

Kester, et., al., observó que las empresas tienden a comprometer fondos en las inversiones mas pronto que tarde, a pesar de la posibilidad de diferir en el tiempo dicho compromiso. La razón reside en que una opción es mas valiosa cuando se posee en exclusiva que cuando es compartida porque los competidores pueden replicar las inversiones de la empresa consiguiendo con ello la reducción de la rentabilidad de la decisión de inversión. Así que este último se realizará antes de la fecha de vencimiento de la opción siempre que el coste de su post-posición supere al valor sacrificado al ejercer la opción de inversión anticipadamente. Esto suele ocurrir cuando: las opciones son compartidas; el VAN de la decisión de inversión es alta; los niveles de riesgo y de tipo de interés son bajos y la competencia es alta.

En conclusión, para Mascareñas et., al., las opciones compartidas suele carecer de valor y las únicas que interesan son las opciones en exclusiva, lo malo es que estas últimas no abundan tanto como se quisieran, porque para ello será necesario tener barreras de entrada (por ejemplo, patentes), o ventajas competitivas.

Caso de una opción de diferir la decisión de inversión.

Una compañía minera se planteo cuanto se debía ofrecer por rentar un terreno susceptible de convertirse en una explotación minera de cobre, su equipo directivo extrapoló el precio de dicho 
mineral durante el horizonte temporal de la inversión, además, hizo lo mismo con los costos de extracción, con los impuestos y estimó la cantidad de cobre que podía extraerse de la mina, con todo ello calculo el valor actual de los flujos de caja implicados en el desarrollo de la explotación minera y en la venta de cobre. Concluyó que la renta valía como mucho 17 millones de soles. El equipo directivo de la empresa era consiente de que la fluctuación del precio de cobre podría ser bastante grande. Como su precio actual estaba próximo al umbral de rentabilidad de la decisión de inversión, las proyecciones de ingresos eran muy sensibles con respecto a las variaciones del precio en el futuro.

La empresa se dio cuenta de que al pagar la renta estaría adquiriendo una opción para diferir la apertura de la mina hasta el instante en que el precio del mineral ascendiese lo suficiente para que la operaciones resultará económicamente beneficiosa. Esta opción tomo un valor de 16 millones de soles, casi lo mismo que la mina si se ponía en explotación inmediatamente. Así el valor máximo del alquiler pasaba a ser 32 millones de soles. La empresa ofreció 20 millones de soles al propietario del terreno por alquilarselo, espero hasta que el precio se situó a un nivel adecuado y obtuvo una buena ganancia con la operación.

\section{REFERENCIAS}

1. Aizenman, J. y Marión, N. Volatility and Investment: Interpreting Evidense from Developing Countries, Rev. Económica No. 262, London, 1999.
2. Assael, Héctor, Desarrollo Financiero y Crecimiento Productivo en América Latina, Santiago de Chile, 2004.

3. Brealey, Richard y Myers, Stewart, Fundamentos de Financiación Empresarial, Mc Grw Hill, Madrid 1993.

4. Diez, L. y Mascareñas, J. Ingenieria Financiera, Mc Graw Hill, Madrid, 1994.

5. Ffrench Davis, R. yH. Reisen (eds.), Flujos de Capital e Inversión productiva: Lecciones para America Latina, Mc Journal of Financial an Quantitative Analysis, December 1983.

6. Rivas, G., Opciones de la Banca de Desarrollo en Chile: El «Convidado de Piedra" del Sistema Financiero Chileno, Santiago de Chile, 2003.

7. Ryd, Gunilla, Estados Unidos: ¿Una Nueva Economía o más de los mismo?, Santiago de Chile, 2003.

8. Salazar, J.C. y B.A. Montenegro, La Financiación de las Iniciativas Empresariales de Innovación Tecnologica en Colombia, Bogotá, 2003.

9. Trigeorgis, Lenos y Mason, Scott, Valoración de la Flexibilidad Futura en las Decisiones de Inversión, Praeger, 1995

10. Trigeorgis, Lenos y Mason, Scott, Valoración de la Flexibilidad Futura en las decisiones de Inversión, Revista Análisis Financiero No 54, 1991. 